\title{
Anticoncepcional oral: efeitos colaterais e a sua relação com a trombose venosa
}

\author{
Oral contraceptive: side effects and its relationship with venous thrombosis \\ Anticonceptivo oral: efectos secundarios y su relación con la trombosis venosa
}

Sabrina Luíza Ames da Cruz ORCID: https://orcid.org/0000-0002-4536-9392 Faculdade de Palmas, Brasil

E-mail: sabrinaames1999@gmail.com

Daniel dos Santos Bottega ORCID: https://orcid.org/0000-0003-2562-0065 Faculdade de Palmas, Brasil

E-mail: danielbottega012@gmail.com

Maykon Jhuly Martins de Paiva ORCID: https://orcid.org/0000-0002-6228-4550 Faculdade de Palmas, Brasil

E-mail: maykonjhuly@ hotmail.com

\begin{abstract}
Resumo
A trombose venosa profunda é uma doença que acomete milhões de pessoas ao redor do mundo todos os anos. Para o sexo feminino, existe um agente de exposição constante e muito frequente que são os anticoncepcionais orais, esses medicamentos são utilizados para evitar a gravidez, e em alguns casos para tratar doenças de cunho hormonal e que acometem apenas mulheres. É descrito na literatura que os anticoncepcionais orais causam esses riscos pois são compostos por hormônios sintéticos como: progesterona e estrógeno, e eles afetam a coagulação sanguínea. O objetivo da pesquisa é avaliar como ocorre as reações ao anticoncepcional hormonal oral no corpo da mulher e sua relação com a trombose venosa. O presente trabalho trata-se de uma revisão bibliográfica nas bases de dados Scielo, Google Acadêmico, Lilacs e BVS com foco nas correlações entre o uso do anticoncepcional e a trombose venosa profunda, estudando a hemostasia e os efeitos farmacológicos dos anticoncepcionais hormonais orais na cascata de coagulação. Conclui-se que os contraceptivos hormonais podem ter como efeito adverso a trombose venosa, pois os hormônios utilizados em sua formulação acarretam mudanças na cascata de coagulação e inibem fatores que auxiliam o organismo a evitar a hipercoagulabilidade.
\end{abstract}

Palavras-chave: Hemostasia; Trombose venosa; Anticoncepcional oral.

\begin{abstract}
Deep vein thrombosis is a disease that affects millions of people around the world every year, there are predispositions to this disease in both sexes such as smoking, genetic conditions and factors related to lifestyle. However, for females there is an agent of constant and very frequent exposure, which is oral contraceptives, these drugs are used to prevent pregnancy, and in some cases to treat diseases of hormonal nature and that affect only women. It is described in the literature that oral contraceptives cause these risks because they are composed of synthetic hormones such as progesterone and estrogen, and they affect blood clotting. This is a literature review in the Scielo, Google Scholar, Lilacs and BVS databases focusing on the correlations between contraceptive use and deep vein thrombosis, studying hemostasis and the pharmacological effects of oral contraceptive hormones in the coagulation cascade. It is concluded that contraceptive hormones can have an adverse effect on venous thrombosis, as the hormones used in their matter cause changes in the coagulation cascade and inhibit factors that help the body to avoid hypercoagulability.
\end{abstract}

Keywords: Hemostasis; Deep vein thrombosis; Oral contraceptive.

\section{Resumen}

La trombosis venosa profunda es una enfermedad que afecta a millones de personas en todo el mundo cada año. Para las mujeres existe un agente de exposición constante y muy frecuente, que son los anticonceptivos orales, estos medicamentos se utilizan para prevenir el embarazo, y en algunos casos para tratar enfermedades de carácter hormonal que afectan solo a las mujeres. Se describe en la literatura que los anticonceptivos orales causan estos riesgos porque están compuestos de hormonas sintéticas como la progesterona y el estrógeno, y afectan la coagulación de la sangre. El objetivo general de la investigación es evaluar cómo ocurren las reacciones a los anticonceptivos hormonales orales en el cuerpo de la mujer y su relación con la trombosis venosa. El presente trabajo es una revisión de la literatura em las bases de datos Scielo, Google Academic, Lilacs y BVS, centrándo se en las correlaciones entre el uso de anticonceptivos 
y la trombosis venosa profunda, estudiando la hemostasia y los efectos farmacológicos de los anticonceptivos hormonales orales en la cascada de la coagulación. Se concluye que los anticonceptivos hormonales pueden tener un efecto adverso sobre la trombosis venosa, ya que las hormonas utilizadas en su formulación provocan cambios em la cascada de la coagulación e inhiben factores que ayudan al organismo a evitar la hipercoagulabilidad.

Palabras clave: Hemostasia; Trombosis venosa; Anticonceptivo oral.

\section{Introdução}

O método de contracepção hormonal oral é o mais utilizado pelas mulheres, por conta da facilidade para adquirir o medicamento pelo Sistema Único de Saúde. Os mais conhecidos são as pílulas combinadas, elas possuem progesterona e estrogênio (Reis et al., 2018).

A eficácia do contraceptivo hormonal se dá pela capacidade dele de inibir a ovulação e alterar características no endométrio e muco cervical, evitando a gravidez. Essa capacidade é característica do estrogênio, esse hormônio age no hipotálamo causando a inibição da secreção de GNRH e de modo consequente do FSH e do LH, sendo que esses são fundamentais para que ocorra a ovulação (Silva, Sá \& Toledo, 2019).

A TVP é uma patologia caracterizada pela ausência de circulação sanguínea no tecido causada por um trombo, os números de casos são frequentes e é uma condição grave, o qual a torna um dos motivos mais recorrentes de atendimentos emergenciais, isso faz com que seja uma doença que tem um custo alto, tanto social quanto econômico (Lima, Souza, Dourado, De Oliveira \& Dos Santos, 2019).

No Brasil patologias do sistema circulatório, englobando os tipos de tromboses, ocupam o terceiro lugar quando se leva em consideração a incidência, e no ano que foi realizado a pesquisa cerca de 170 mil pessoas sofriam com o tromboembolismo (Guimarães, 2016).

A hemostasia é a responsável em manter a fluidez sanguínea, sendo o principal agente para que haja chegada de sangue em todos os tecidos. (Guimarães, 2016). Ela também é responsável pela regulação dos fatores de coagulação, pois, a partir desses fatores que vai ser impedido que haja hemorragia em casos de lesão vascular, e ao mesmo tempo impedindo que aconteça uma coagulação intensa demais causando obstrução do vaso sanguíneo (Moreira et al., 2016).

Faz parte dos efeitos adversos dos anticoncepcionais orais o risco aumentado da ocorrência de trombose venosa, pois seus componentes causam alterações na cascata de coagulação, comprometendo a funcionalidade da hemostasia e inibindo fatores de coagulação naturais (Braga \& Vieira, 2013; Morais, Santos \& Carvalho, 2019).

O uso de contraceptivos hormonais foi relacionado ao aumento de risco de TVP. Pois os componentes das pílulas hormonais combinadas reagem com as camadas que compõem os vasos, pois elas possuem receptores de estrogênio e progesterona, e isso torna o endotélio reativo aos componentes do sangue, causando também a inibição dos fatores de coagulação natural além de estimular fatores de hipercoagulabilidade (Da Silva \& Hayd, 2017).

Estudos indicam que o estrogênio utilizado nos contraceptivos combinados interfere na hemostasia por conta das alterações que ela sofre e por esse motivo aumentam as chances de desenvolvimento de tromboembolismo arterial e venoso, ele é capaz de causar desordens endoteliais alterando o sistema de coagulação. Em contrapartida, os progestagênios usados de forma isolada não demonstraram alterar fatores de riscos para o desenvolvimento de tromboses (Braga \& Vieira, 2013; Batista, Lima \& Oliveira-Kumakura, 2021).

Este estudo visa explanar como ocorre as reações ao anticoncepcional hormonal oral no corpo da mulher e sua relação com a trombose venosa. 


\section{Metodologia}

A metodologia adotada para a elaboração desse trabalho foi a revisão bibliográfica com o levantamento de dados nas plataformas Scientific Eletronic Library (SCIELO), Literatura latino-americano em Ciências da Saúde (LILACS), Google acadêmico, além do banco de dados da Biblioteca Virtual em Saúde (BVS) já que estas permitem buscas simultâneas em fontes nacionais e internacionais.

Para o acesso dos artigos selecionados foram utilizados os seguintes descritores: anticoncepcional oral, trombose venosa profunda e hemostasia. Os fatores de inclusão foram: artigos em português e inglês que estivessem dentro das plataformas selecionadas, com publicação entre os anos de 2013 e 2021. Foram utilizadas como critérios de exclusão a leitura do resumo e da introdução para a verificação de informações, objetivos em comum com essa pesquisa.

\section{Resultados e Discussões}

Foram encontrados 26 artigos que possuem informações sobre anticoncepcionais hormonais orais, trombose venosa, hemostasia e a relação entre esses fatores, 2 foram excluídos por conterem informações repetidas.

Figura 1 - Processo de seleção utilizado para elaborar o estudo de revisão bibliográfica, fluxograma feito baseado nas orientações PRISMA.

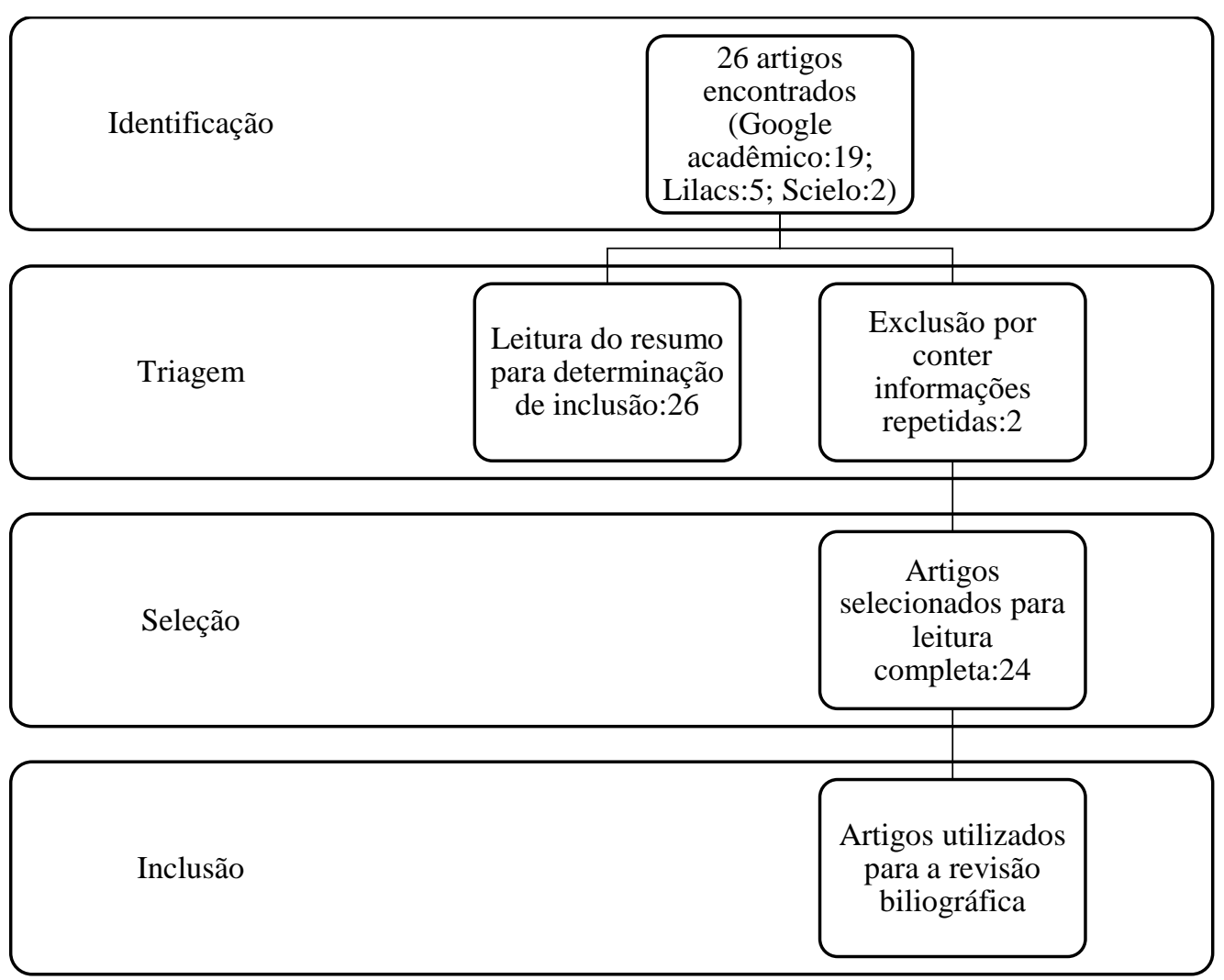

Fonte: Autores (2021).

A quantidade de artigos utilizados, ano de publicação, língua de publicação e os principais resultados de cada artigo estão explicitados nos quadros abaixo: 
Quadro 1 - Quantidade de artigos publicados entre os anos de 2013 e 2021.

\begin{tabular}{|c|c|}
\hline Ano da publicação & Quantidade de artigos \\
\hline 2013 & 1 \\
\hline 2014 & 1 \\
\hline 2015 & 2 \\
\hline 2016 & 3 \\
\hline 2017 & 4 \\
\hline 2018 & 4 \\
\hline 2019 & 7 \\
\hline 2021 & 2 \\
\hline
\end{tabular}

Fonte: Autores (2021).

Quadro 2 - Informações principais dos artigos selecionados.

\begin{tabular}{|c|c|c|}
\hline Título do artigo & Resultados & $\begin{array}{l}\text { Língua de } \\
\text { publicação }\end{array}$ \\
\hline $\begin{array}{l}\text { A eficácia do rastreio de trombofilias antes da } \\
\text { prescrição de métodos contraceptivos. }\end{array}$ & $\begin{array}{l}\text { Não houveram resultados satisfatórios para que exista } \\
\text { indicação de rastreio antes da prescrição de anticoncepcionais } \\
\text { orais. }\end{array}$ & Português \\
\hline $\begin{array}{l}\text { Anticoncepcionais: Relação com trombose venosa } \\
\text { profunda. }\end{array}$ & $\begin{array}{l}\text { Ratificou a necessidade de acompanhamento médico } \\
\text { especializado para a prescrição. }\end{array}$ & Português \\
\hline $\begin{array}{l}\text { A relação entre o uso de anticonconcepcionais } \\
\text { orais e a ocorrência de trombose }\end{array}$ & $\begin{array}{l}\text { Conclui-se que os anticoncepcionais orais interferem na } \\
\text { cascata de coagulação aumentando o risco de trombose } \\
\text { venosa. }\end{array}$ & Português \\
\hline $\begin{array}{l}\text { A relação entre o uso da pílula anticoncepcional e } \\
\text { o desenvolvimento de trombose no Brasil. }\end{array}$ & $\begin{array}{l}\text { Resultados indicaram a necessidade do acompanhamento } \\
\text { médico especializado na prescrição do medicamento. }\end{array}$ & Português \\
\hline $\begin{array}{l}\text { Avaliação do uso de anticoncepcional oral } \\
\text { combinado como fator de risco para o } \\
\text { desenvolvimento de trombose em mulheres jovens } \\
\text { da cidade de Patos. }\end{array}$ & $\begin{array}{l}\text { Apresentou as alterações que ocorrem nos testes para } \\
\text { verificação de coagulação e a necessidade } \\
\text { acompanhamento médico como medida profilática. }\end{array}$ & Português \\
\hline Causes of venous thrombosis & $\begin{array}{l}\text { O artigo abordou as principais causas, citando utilização de } \\
\text { hormônios como fator de risco para o desenvolvimento de } \\
\text { trombose. }\end{array}$ & Inglês \\
\hline Contracepção hormonal e tromboembolismo & $\begin{array}{l}\text { Ressalta a necessidade de avaliação medica criteriosa no } \\
\text { momento da prescrição. }\end{array}$ & Português \\
\hline Farmacoterapia da anticoagulação & $\begin{array}{l}\text { Expos os principais fármacos anticoagulantes, benefícios, } \\
\text { impedimentos por sua forma farmacêuticas e fármacos } \\
\text { alternativos que apresentam bons resultados. }\end{array}$ & Português \\
\hline $\begin{array}{l}\text { Influência de contraceptivos hormonais sobre } \\
\text { indicadores de homeostase de zinco e de turnover } \\
\text { ósseo em mulheres adultas jovens }\end{array}$ & $\begin{array}{l}\text { Indica a diminuição de níveis séricos de zinco em mulheres } \\
\text { que utilizam anticoncepcional oral. }\end{array}$ & Português \\
\hline $\begin{array}{l}\text { Interações medicamentosas com contraceptivos } \\
\text { hormonais orais }\end{array}$ & $\begin{array}{l}\text { Constata as interferências de outros medicamentos na } \\
\text { efetividade da pílula anticoncepcional. }\end{array}$ & Português \\
\hline $\begin{array}{l}\text { Métodos contraceptivos e a prevalência de } \\
\text { mulheres adultas e jovens com risco de trombose, } \\
\text { no Campus Centro Universitário do Distrito } \\
\text { Federal-UDF }\end{array}$ & $\begin{array}{l}\text { Caracteriza os riscos de trombose que o anticoncepcional oral } \\
\text { causa. }\end{array}$ & Português \\
\hline $\begin{array}{l}\text { Mulheres portadoras de trombofilia: Entre } \\
\text { narrativas da reprodução assistida mandatória e da } \\
\text { tragédia relacionada aos anticoncepcionais } \\
\text { hormonais. }\end{array}$ & $\begin{array}{l}\text { Reitera a necessidade da avaliação medica criteriosa e traz } \\
\text { dados sobre ocorrência de trombose em mulheres trombofilias. }\end{array}$ & Português \\
\hline $\begin{array}{l}\text { O uso da pílula anticoncepcional e as alterações } \\
\text { das principais vias metabólicas }\end{array}$ & $\begin{array}{l}\text { Expõe as alterações metabólicas causadas pelo } \\
\text { anticoncepcional oral, inclusive as alterações na cascata de } \\
\text { coagulação responsável pela regulação para que não haja } \\
\text { trombose. }\end{array}$ & Português \\
\hline
\end{tabular}




\begin{tabular}{|c|c|c|}
\hline $\begin{array}{l}\text { Profilaxia de tromboembolismo venoso, podemos } \\
\text { fazer melhor? Perfil de risco e profilaxia de } \\
\text { tromboembolismo venoso em Hospital } \\
\text { Universitário do interior do Estado de São Paulo. }\end{array}$ & $\begin{array}{l}\text { A pesquisa aborda métodos de profilaxia de trombose, } \\
\text { constatando os erros que acontecem na prescrição do } \\
\text { tratamento correto. }\end{array}$ & Português \\
\hline $\begin{array}{l}\text { Quadro clínico de mulheres acometidas por } \\
\text { acidente vascular cerebral em uso de } \\
\text { anticoncepcionais hormonais. }\end{array}$ & $\begin{array}{l}\text { Após a análise dos estudos foi identificado que usuárias de } \\
\text { anticoncepcional hormonal apresentaram maior taxa de } \\
\text { mortalidade e risco aumentado de desenvolver outra trombose. }\end{array}$ & Português \\
\hline $\begin{array}{l}\text { Risco de trombose relacionada ao uso de } \\
\text { hormonas e evidenciada pela quebra de } \\
\text { hemostasia: Uma breve revisão }\end{array}$ & $\begin{array}{l}\text { Relacionou os hormônios utilizados nas pílulas } \\
\text { anticoncepcionais com a trombose, indicando que o estrogênio } \\
\text { é o hormônio que causa alterações na coagulação pois interage } \\
\text { com os receptores endoteliais. }\end{array}$ & Português \\
\hline $\begin{array}{l}\text { Risk prediction of developing venous thrombosis } \\
\text { in combined oral contraceptive users }\end{array}$ & $\begin{array}{l}\text { O estudo foi capaz de identificar dois novos marcadores } \\
\text { genéticos que indicam aumento de risco de trombose em } \\
\text { usuárias de anticoncepcional oral combinado. }\end{array}$ & Inglês \\
\hline $\begin{array}{l}\text { Tabagismo e uso de anticoncepcionais orais } \\
\text { relacionados a fenômenos tromboembólicos: } \\
\text { relato de caso e revisão de literatura }\end{array}$ & $\begin{array}{l}\text { Traz resultados positivos sobre a relação entre } \\
\text { anticoncepcionais orais e o tabagismo, pois juntos } \\
\text { potencializam a capacidade do corpo de produzir coágulos. }\end{array}$ & Português \\
\hline $\begin{array}{l}\text { Trombose venosa profunda associada ao uso de } \\
\text { anticoncepcional: relato de caso }\end{array}$ & $\begin{array}{l}\text { O relato indicou que a paciente que fazia o uso de } \\
\text { anticoncepcional oral era portadora de trombofilia Fator V de } \\
\text { Leiden e possuía caso de trombose na família. }\end{array}$ & Português \\
\hline $\begin{array}{l}\text { Trombose associada ao uso de contraceptivo } \\
\text { hormonal oral: Revisão de literatura }\end{array}$ & $\begin{array}{l}\text { Indicou que os contraceptivos hormonais orais de terceira } \\
\text { geração são os que mais causam riscos para ocasionalmente de } \\
\text { trombose venosa. }\end{array}$ & Português \\
\hline $\begin{array}{l}\text { Trombose venosa profunda num membro superior } \\
\text { em mulher a fazer anticoncepcional oral com } \\
\text { trombofilia hereditária- Factor V Leiden }\end{array}$ & $\begin{array}{l}\text { Explicitou a contribuição da mutação genética Fator V de } \\
\text { Leiden para o risco aumentado de desenvolvimento de evento } \\
\text { trombótico. }\end{array}$ & Português \\
\hline $\begin{array}{l}\text { Trombose venosa profunda como reação adversa } \\
\text { do uso contínuo de anticoncepcionais orais. }\end{array}$ & $\begin{array}{l}\text { Reforça o fato que uso de anticoncepcional hormonal oral } \\
\text { pode ocasionar uma trombose venosa por conta das alterações } \\
\text { que o medicamento causa na hemostasia, podendo causar } \\
\text { patologias graves como trombose cerebral. }\end{array}$ & Português \\
\hline $\begin{array}{l}\text { Tromboembolismo venoso relacionado ao uso } \\
\text { frequente de anticoncepcionais orais combinados. }\end{array}$ & $\begin{array}{l}\text { A pesquisa indicou que que é necessário avaliar qual o melhor } \\
\text { método a ser utilizado na contracepção por conta dos riscos } \\
\text { que os anticoncepcionais orais causam a regulação de } \\
\text { coagulação do organismo. }\end{array}$ & Português \\
\hline $\begin{array}{l}\text { Utilização de contraceptivos orais contendo } \\
\text { etinilestradiol e a ocorrência de trombose venosa } \\
\text { profunda em membros inferiores }\end{array}$ & $\begin{array}{l}\text { Evidenciou os efeitos causados pelo etinilestradiol na cascata } \\
\text { de coagulação, causando o risco aumentado de trombose }\end{array}$ & Português \\
\hline
\end{tabular}

Fonte: Autores (2021).

Os artigos contidos nos quadros possuem informações sobre a farmacologia dos anticoncepcionais orais, ocorrência de trombose e hemostasia do organismo resultando em conclusões sobre os efeitos dos anticoncepcionais na cascata de coagulação relacionado com a sua farmacologia e a mudança na hemostasia.

\section{Hemostasia}

A hemostasia se dá por mecanismos que garantem que haja fluidez do sangue nos vasos. Esse processo fisiológico mantém o sangue em movimento nos vasos sanguíneos com o propósito de evitar a formação de coágulos e consequentemente eventos hemorrágicos e/ou tromboembolísticos (Ferreira, Dávila \& Safatle, 2019).

Sempre que acontece alguma lesão a algum vaso sanguíneo o sistema hemostático age com seus mecanismos de contração do vaso, formação do tampão plaquetário, formação de um coágulo sanguíneo e o crescimento do tecido fibroso. Ele 
está diretamente relacionado ao número de plaquetas, resistência e contratilidade dos vasos e um processo de coagulação adequado (Sousa \& Álvares, 2018).

Esses processos ocorrem na chamada "cascata de coagulação" estimulando assim, a produção de trombina, a qual converte fibrinogênio solúvel em fibrina solúvel. Ademais, há ampliação dos fatores de coagulação (fibrinogênio, VII, VIII, IX, X, XII e XIII) e diminuição dos inibidores naturais da coagulação (proteína S e antitrombina), o que implica em um efeito prócoagulante leve (Ferreira, Dávila \& Safatle, 2019).

Existem, no sistema hemostático, anticoagulantes naturais que fazem a regulação e o bloqueio da cascata de coagulação, consequente da quebra proteolítica dos fatores de coagulação constituído pela antitrombina III e as proteínas C e S (Maia, 2015).

\section{Anticoncepcional oral}

Os anticoncepcionais orais são utilizados no mundo inteiro a mais de três décadas, e por consequência da sua composição as usuárias do medicamento passam por alguns efeitos colaterais como: náuseas, fortes dores de cabeça, dor de estômago, ganho de peso, inchaço, além de aumento de vasos em membros inferiores. No início da comercialização as doses hormonais eram muito altas e os seus efeitos adversos prejudicavam de forma intensa o dia a dia das mulheres' (Moreira et al., 2016).

A primeira pílula continha cerca de 5.000 microgramas de progesterona e 150 microgramas de estrogênio, o que é um percentual muito alto em comparação com uma dose encontrada nas pílulas atuais. Atualmente, encontra-se no mercado duas categorias de contracepção hormonal, métodos que combinam estrogênio, progesterona e os exclusivos de progesterona (Moreira et al., 2016).

O contraceptivo hormonal é composto por hormônios sintéticos, geralmente estrogênio e progesterona, e possuem o intuito de impedir a ovulação evitando, assim, a gravidez. Desde o início de sua formulação já passou por algumas mudanças sendo divididos em quatro gerações sendo a última a com menor concentração hormonal. São divididos em três tipos: monofásicos, bifásicos e trifásicos. Os monofásicos possuem a mesma concentração hormonal em todos os comprimidos da cartela, os bifásicos e trifásicos são divididos por duas e três concentrações diferentes ao longo do uso mensal (Ferreira, Dávila \& Safatle, 2019).

Os anticoncepcionais orais realizam suas funções alterando o sistema neuroendócrino, intervindo no mecanismo de estimulação ovariano que efetua um bloqueio gonadotrófico e com isso impede a ovulação (Moreira et al.. 2016). Agem sobre os hormônios que desencadeiam a ovulação, mantendo os níveis constantes de progesterona e estrogênio, inibindo a secreção hipofisária de hormônio luteinizante (LH) e o hormônio folículo estimulante (FSH) do mecanismo de "feedback", mantendo os óvulos inativados e impedindo a ovulação. Assim o fármaco reduz a chance de haver ovulação e fecundação no período da utilização do medicamento (Sousa \& Álvares, 2018).

Os contraceptivos hormonais orais atuam inibindo a ovulação mediante o bloqueio seletivo da hipófise e do hipotálamo via retroalimentação negativa, não havendo assim liberação de LH, FSH e gonadotrofina, fazendo com que o muco cervical fique espesso e aumente a dificuldade da passagem dos espermatozoides, além de provocar alterações no endométrio, na secreção e na motilidade das tubas uterinas, dificultando dessa forma a gravidez (Silva, Santana, Nunes, Santos \& Terra).

Os anticoncepcionais orais combinados (estrógenos + progestágenos) apresentam eficácia de aproximadamente 99,9\%, já as minipílulas (progestágenos isolados) apresentam aproximadamente apenas 99\% de eficácia, porém os efeitos adversos dos anticoncepcionais simples são pouco citados e seu uso não tem relação com a elevação do risco para doenças malignas (Silva, 2018; Magalhaes \& Morato, 2018; Silva, Thomaz, Melo \& Martins 2017). 


\section{Efeitos colaterais do anticoncepcional no corpo da mulher}

É certo que uso dos contraceptivos são beneficiais, pois diminuem o fluxo menstrual, a intensidade das cólicas, tratam cistos e, claro, evitam a gravidez indesejada promovendo uma maior possibilidade de planejamento familiar. Porém, seu uso traz riscos e por conta deles é necessária que haja cautela e acompanhamento profissional durante todo o período de uso desses medicamentos (Maia, 2015).

Alguns estudos avaliam os riscos que esse medicamento traz a saúde da mulher, foi realizada uma pesquisa que aponta que mulheres que fazem o uso de contraceptivos hormonais possuem o nível sérico de zinco reduzido, além de afetar a distribuição do mesmo, fazendo com que a longo prazo essas pessoas tenham pico de massa óssea menor que o normal (Simões, Zapata \& Donangelo, 2015).

O risco mais preocupante que os anticoncepcionais orais causam a saúde da mulher são as chances aumentadas de tromboses venosas. Isso ocorre por conta dos hormônios sintéticos que causam a inibição de alguns fatores de anticoagulação natural do organismo, como: inibição da proteína $\mathrm{S}$ e antitrombina, esse fenômeno é causado pelo estrogênio (Ferreira \& Da Paixão, 2021).

\section{Trombose venosa e trombose venosa profunda}

A trombose venosa é uma patologia causada pelo bloqueio de circulação sanguínea por um coágulo, impedindo a oxigenação do tecido afetado, são mais comuns nos membros inferiores, mas podem ocorrer em várias regiões do corpo causando acidente vascular cerebral, infarto do miocárdio, tromboembolia pulmonar entre outras (Sousa \& Álvares, 2018).

A trombose venosa profunda é definida pela produção de trombos no interior de veias mais profundas e densas. Existem fatores que causam essa alteração na hemostasia e uma delas é o uso de contraceptivo hormonal oral (Morais, Santos \& Carvalho, 2019).

Essas patologias são caracterizadas como graves pois tem uma morbimortalidade alta. Qualquer pessoa está suscetível a ter um evento trombótico, tendo o número de casos aumentados quando a idade é maior e quando há uma predisposição para tais eventos. A probabilidade de uma de uma mulher jovem desenvolver uma trombose venosa profunda quando utiliza anticoncepcionais orais é de duas a seis vezes maior defrontado a uma mulher jovem que não faz uso de anticoncepcionais orais (Sousa \& Álvares, 2018; Rosendaal, 2016).

O diagnóstico de trombose venosa profunda é feito através da anamnese dos sintomas em conjunto com exames de imagem, como o USG Doppler e venografia além de exames de sangue como o “dímero D” sendo um elemento resultante da degradação de fibrina, sendo uma proteína envolvida com a formação de coágulos, portanto quando ocorre uma alteração desse fator significa que existe uma circulação muito grande de dímero D no sangue o que é indicativo de TVP (Sousa \& Álvares, 2018).

O tratamento da trombose é realizado segundo a necessidade clínica de cada indivíduo, pois existem grandes variações de fármacos anticoagulantes que se diferenciam nas características farmacocinéticas e farmacológicas, em caso de internação hospitalar a equipe médica é responsável pela prescrição do tratamento e profilaxia após o evento (Rodrigues, 2014; Curtarelli et al., 2018).

\section{O uso do anticoncepcional e a trombose venosa profunda}

Um importante fator de risco para a trombose venosa profunda é o uso dos anticoncepcionais orais combinados, estudos indicam que os contraceptivos hormonais têm sido responsáveis de 9 a 18\% dos casos de TV em mulheres (Sena \& Gonçalves, 2019; McDaid et al.,2017). 
Os estrogênios estão mais associados a trombose venosa profunda por serem capazes de inibir fatores importantes da anticoagulação natural. A OMS postula que não deve se usar anticoncepcionais orais combinados em mulheres que possuam síndrome trombogênica reconhecida (Maia, 2015).

Existem estudos que apontam que o tabagismo e o álcool, combinados com um estilo de vida sedentário acaba acarretando um maior número de casos de trombose venosa profunda em mulheres que fazem o uso de anticoncepcional oral, deve-se levar em consideração as doenças hematológicas chamadas de trombofilias que são de cunho genético e hereditário e também causa um aumento de chance de trombose venosa profunda. A trombofilia hereditária mais comum é o fator V de Leiden, com prevalência que varia de 2 a $7 \%$ na população europeia e é caracterizada como a mais elevada nessa região (Callai et al., 2017; De Moraes, Gama Alves \& Castiel, 2019).

Os indivíduos que possuem o fator $\mathrm{V}$ de Leiden apresentam um risco de 20 a 50 vezes maior de desenvolver algum evento trombótico do que indivíduos que não são homozigotos para essa condição. Portanto mulheres que fazem o uso de anticoncepcional oral e com fator V de Leiden tem o risco acrescido de desenvolver fenômenos trombóticos (Maia, 2015).

Em relação ao tabagismo, entende-se que as substâncias encontradas no cigarro fazem a indução de um estado prótrombótico, por conta da estimulação plaquetária, e quando interage com o anticoncepcional oral causa uma elevação da ameaça a eventos trombogênicos, e isso expõe uma atribuição significativa para o acontecimento da comorbidade na mulher (Callai $e t$ al., 2017).

É importante entender o que leva o anticoncepcional hormonal oral ser um risco para a TV. Os hormônios sintéticos (estrogênio e progesterona) que compõem esse medicamento possuem interação com os vasos sanguíneos, pois existem receptores desses hormônios nas camadas dos vasos (Sousa \& Álvares, 2018).

Nas últimas décadas o estrogênio foi relacionado ao aumento do risco de trombose venosa e por conta disso, sua dose foi sendo reduzida. No entanto, estudos apontam que os novos progestágenos usados nos contraceptivos hormonais orais não conseguem reduzir o risco de trombose, inclusive estão relacionados ao aumento dos riscos de tromboembolismo venoso e arterial (Callai et al., 2017).

A correlação entre COC e a trombose venosa ocorre porque o estrogênio atua como $m$ ativador dos fatores de coagulação (fibrinogênio, II, VII, VIII, IX, X e XI), eleva a síntese de trombina, altera a cascata de coagulação além de inibir agentes coagulantes naturais, sendo eles a proteína C reativa e a proteína S (Guimarães, 2016; Sousa \& Álvares, 2018).

Essa inibição favorece a ampliação da hipercoagulabilidade, pois essas proteínas agem de maneira a inibir a coagulação, sendo um anticoagulante endógeno (Proteína $\mathrm{C}$ reativa) e agindo como um cofator não enzimático pois potencializa essas reações (Proteína S) (Sousa \& Álvares, 2018).

Também está descrito que os contraceptivos hormonais alteram a viscosidade sanguínea, a parede vascular. Um estudo cruzado revelou que outro importante fator de coagulação natural, o inibidor da via tecidual, também estava em níveis reduzidos em mulheres que fazem o uso de anticoncepcional hormonal oral, provocando um maior risco trombótico (Guimarães, 2016).

É constatado que há uma vasodilatação induzida pelo progestagênio, essa mudança do tônus vascular pode causar uma diminuição da velocidade do fluxo sanguíneo, o que vai resultar em uma maior estase além do aumento do contato das plaquetas com a parede vascular, aumentando a possibilidade de uma trombose venosa (Guimarães, 2016).

\section{Possíveis soluções para evitar eventos trombóticos}

Estudos apontam que a trombose associada ao anticoncepcional combinado está ligada a dose dependente de estrogênio, ou seja, quanto maior a dose maior o risco de TV. Existe no mercado um anticoncepcional oral que possui uma carga hormonal muito pequena, são as chamadas minipílulas sendo indicadas para puérperas e lactantes assim como para mulheres com histórico 
de eventos trombóticos. Esse é menos perigoso exatamente por sua carga hormonal ser muito baixa e ser composto exclusivamente por progesterona que é um hormônio sintético mais seguro (Morais, Santos \& Carvalho, 2019).

Há formas e apresentações diferentes dos contraceptivos, seja ele por barreira ou com mecanismo diferente como o DIU, é prudente que mulheres com histórico de trombose ou complicação causada por anticoncepcionais hormonais orais optem por um método diferente e que não cause um risco a sua saúde.

Os anticoagulantes orais mais utilizados são Rivaroxabana e Varfarina com o mesmo intuito de agir como profiláticos para formação de novos trombos. Após um evento trombótico é de suma importância um acompanhamento médico e a suspensão do uso do contraceptivo hormonal oral e, se possível uma investigação genética a cerca desse problema, há relatos que após evento trombótico o novo método anticoncepcional adotado foi o preservativo masculino (Magalhaes \& Morato, 2018; Sousa \& Álvares, 2018, Maia, 2015).

\section{Considerações Finais}

Ao analisar os trabalhos científicos acerca do assunto se torna explicito todas as complicações que esses medicamentos causam ao organismo, além de aumentar significativamente os riscos para uma trombose venosa profunda. Contudo, se faz necessário entender que o anticoncepcional oral é o contraceptivo mais acessível e mais utilizado no Brasil, portanto, é extremamente necessário que a mesma política pública que o distribui se torne responsável pelo total acompanhamento da mulher durante todo o seu uso, com exames e frequentes consultas com médicos, sempre explicitando o risco que este medicamento traz a saúde da paciente.

Sabe-se que existem formas alternativas de contracepção seja por barreira física como a camisinha ou os intrauterinos como o DIU de cobre que já é disponibilizado pelo SUS, a ampliação da implantação do diu iria diminuir o número de mulheres utilizando o anticoncepcional oral, diminuindo assim os riscos à saúde delas que optam em obter um melhor planejamento familiar. Respeitar a decisão da mulher, em qualquer hipótese de contracepção, se torna o principal objetivo acerca do assunto, portanto é de suma importância que ela seja informada de todas as opções que possui, para que, de forma consciente escolha a que for mais conveniente ao seu estilo de vida e seu estado de saúde.

As implicações clinicas deste estudo apontaram os riscos da contracepção feita através da pílula anticoncepcional que contêm estrogênio, dando ênfase a trombose venosa como efeito adverso grave decorrente do uso desse medicamento, houveram limitações em relação a obtenção de dados detalhados sobre a progesterona e modificações da hemostasia causadas por ela, abrindo uma lacuna e a necessidade de estudos sobre o assunto.

\section{Referências}

Batista, L. M. O. S., Lima, M. H. de M., \& Oliveira-Kumakura, A. R. de S. Quadro clínico de mulheres acometidas por acidente vascular cerebral em uso de anticoncepcionais hormonais. Research, Society and Development, 10(8), e39210817308, 10.33448/rsd-v10i8.17308. https://rsdjournal.org/index.php/rsd/article/view/17308

Braga, G. C., \& Vieira, C. S. (2013). Contracepção hormonal e tromboembolismo. Revista Brasília Médica, 50(1), 58-62. http://rbm.org.br/details/85/ptbr/contracepcao-hormonal-e-tromboembolismo

Callai, T., Daronco, F., Konrad, N. L., Wichmann, J. F., Costa, F., \& Prezzi, S. H. (2017). Tabagismo e uso de anticoncepcionais orais relacionados a fenômenos tromboembólicos: relato de caso e revisão de literatura. Reprodução \& Climatério, 32(2), 138-144. https://www.sciencedirect.com/science/article/pii/s1413208716300759

Curtarelli A, Silva L. P. C, Camargo P. A. B., et al. Profilaxia de tromboembolismo venoso, podemos fazer melhor? Perfil de risco e profilaxia de tromboembolismo venoso em hospital universitário do interior do estado de São Paulo. 18: e20180040. https://www.jvascbras.org/article/10.1590/16775449.004018/pdf/jvb-18-e20180040.pdf

Da Silva, K. R., \& Hayd, R. L. N. (2017) Risco de trombose relacionada ao uso de hormonas e evidenciada pela quebra de hemostasia: uma breve revisão. http://mensagitat.org/data/documents/v-12-p-11-15.pdf 
de Moraes, D. R., da Gama Alves, A. P. P., \& Castiel, L. D. (2019). Mulheres portadoras de trombofilia: entre narrativas da reprodução assistida mandatória e da tragédia relacionada aos anticoncepcionais hormonais. Revista Mundaú, (6), 103-121. https://www.seer.ufal.br/index.php/revistamundau/article/view/6855

Ferreira, B. B. R., \& da Paixão, J. A. (2021). A relação entre o uso da pílula anticoncepcional e o desenvolvimento da trombose venosa profunda no Brasil. Revista Artigos. Com, 29, e7766-e7766. https://acervomais.com.br/index.php/artigos/article/view/7766

Ferreira, L. F., D’avila, A. M. F. C., \& Safatle, G. C. B. (2019). O uso da pílula anticoncepcional e as alterações das principais vias metabólicas. Femina. [Internet], 47(7), 426-32. https://docs.bvsalud.org/biblioref/2019/12/1046533/femina-2019-477-426-432.pdf

Guimarães, M. A. Trombose associada ao uso de contraceptivo hormonal oral: revisão de literatura. 2016. 34 f. Monografia (Graduação) - Faculdade de Ciências da Educação e Saúde, Centro Universitário de Brasília, Brasília, 2016. https://repositorio.uniceub.br/jspui/bitstream/235/9053/1/21352214.pdf

Lima, A. B. D. S., Souza, J. C. D. S., Dourado, M. S., De Oliveira, M. C. S., \& Dos Santos, J. B. C. (2019). Anticoncepcionais: relação com trombose venosa profunda. Anais Eletrônico CIC, 17(1). 2019. http://www.fasb.edu.br/revista/index.php/cic/article/view/401

Magalhaes, A. V. P. De, \& Morato, C. B. A. (2018). Avaliação do uso de anticoncepcional oral combinado como fator de risco para o desenvolvimento de trombose em mulheres jovens da cidade de patos. Caderno de graduação - ciências biológicas e da saúde - unit - Pernambuco, 4(1), 77. https://periodicos.set.edu.br/facipesaude/article/view/6415/3151

Maia, H. O. (2015). Trombose venosa profunda num membro superior em mulher a fazer anticoncepcional oral e com trombofilia hereditária - factor $\mathrm{v}$ leiden. Revista portuguesa de medicina geral e familiar, 31(2), 121-4. https://www.rpmgf.pt/ojs/index.php/rpmgf/article/view/11467

McDaid A, Logette E, Buchillier V, Muriset M, Suchon P, Pache T. D., et al. (2017) Risk prediction of developing venous thrombosis in combined oral contraceptive users. PLoS ONE 12(7): e0182041. https://journals.plos.org/plosone/article?id=10.1371/journal.pone.0182041

Morais, L. X., Santos, L. P., \& Carvalho, I. F. F. R. (2019). Tromboembolismo venoso relacionado ao uso frequente de anticoncepcionais orais combinados. Revista Eletrônica de Ciências Humanas, Saúde e Tecnologia, 8(1), 85-109. https://revista.fasem.edu.br/index.php/fasem/article/view/195

Moreira, F. F. B., Fernandes, M. V. O., Paes, N. F., Netto, R. M. O., Baptista, R. G., \& Souza, J. D. (2016). A eficácia do rastreio de trombofilas antes da prescrição de métodos contaceptivos. BrazilianJournalofSurgeryandClinicalResearch, 15(1), 91-95. <http://www.repositorio.ufop.br/handle/123456789/6609>

Reis, A. L. O, Vasconcelos, J. D. S, Dos Santos, Da Silva, L. C. A, Aredes, L. H. S, Nantes, M. C, Soares, A. L. F, Cesar, J. J. Eler, J. F. De Castro \& Paro, M. \& de Oliveira. (2018). Utilização de contraceptivos orais Contendoetinilestradiol e a ocorrência de trombose venosa profunda em membros inferiores. BrazilianJournalofSurgeryandClinicalResearch - BJSCR.v.23, n. 2, 120-127. https://www.mastereditora.com.br/periodico/20180704_092924.pdf

Rodrigues, D. A. D. S. (2014). Farmacoterapia da anticoagulação (Doctoraldissertation). <https://sapientia.ualg.pt/handle/10400.1/7881

Rosendaal, F.R. Causes of venous thrombosis. Thrombosis J 14, 24 (2016). https://thrombosisjournal.biomedcentral.com/track/pdf/10.1186/s12959-016-0108y.pdf

Sena, C. R. L. D., \& Gonçalves, P. T. T. (2019). Trombose venosa profunda associada ao uso do anticoncepcional oral: relato de caso http://repositorio.saolucas.edu.br:8080/xmlui/handle/123456789/3224

Silva C. S., Sá R, \& Toledo J. Métodos contraceptivos e prevalência de mulheres adultas e jovens com risco de trombose, no campus centro universitário do distrito federal-udf. Revisa. 2019, 8(2):190-7. http://revistafacesa.senaaires.com.br/index.php/revisa/article/view/403/290

Silva, J. E., Santana, K. D. S., Nunes, J. D. S., Santos, J. C. D., \& Terra, A. T. Jr. (2018). A relação entre o uso de anticoncepcionais orais e a ocorrência de trombose. Http://www.faema.edu.br/revistas/index.php/revista-faema/article/view/522

Silva, N. C. S., Martins, S. B. M., Melo, J. A., \& Thomaz, A. P. S. (2017). Interações medicamentosas com contraceptivos hormonais orais. ÚNICA Cadernos Acadêmicos, 3(1). http://co.unicaen.com.br:89/periodicos/index.php/unica/article/view/57

Simões, T. M. R., Zapata, C. L. V., \& Donangelo, C. M. (2015). Influência de contraceptivos hormonais sobre indicadores de homeostase de zinco e de turnover ósseo em mulheres adultas jovens. Revista Brasileira de Ginecologia e Obstetrícia, 37, 402-410. https://www.sc ielo.br/j/rbgo/a/6jwrky9wy6fpcxnhgxcsmpc/?lang=pt\#

Sousa, I. C. D. A. D., \& Álvares, A. D. C. M. (2018). A trombose venosa profunda como reação adversa do uso contínuo de anticoncepcionais orais. Revista de Divulgação Científica Sena Aires, 7(1), 54-65. http://revistafacesa.senaaires.com.br/index.php/revisa/article/view/304 